УДК 624.014

\title{
ОЦЕНКА ВЛИЯНИЯ ЖЁСТКОСТИ УПРУГОГО ЗАЩЕМЛЕНИЯ СТЕРЖНЕЙ И НАЧАЛЬНЫХ НЕСОВЕРШЕНСТВ ФОРМЫ НА ВЕЛИЧИНУ КРИТИЧЕСКОЙ УЗЛОВОЙ НАГРУЗКИ ДЛЯ СЕТЧАТЫХ КУПОЛОВ
}

\author{
К. К. Глушко1, К. А. Глушко
}

${ }^{1}$ K. т. н., старший преподаватель кафредры архитектуры учреждения образования «Брестский государственный технический университет», Брест, Беларусь, e-mail: konstantin.glushko@bk.ru

${ }^{2}$ K. т. н., доцент, доцент кафедры природообустройства учреждения образования «Брестский государственный технический университет», Брест, Беларусь

\section{Реферат}

Рассмотрена задача местной устойчивости стальных сетчатых куполов с упруго-податливым соединением стержней в узлах. Определены зависимости изменения критической узловой нагрузки от податливости узлов. Сформулировано условие местной устойчивости сетчатых куполов.

Ключевые слова: сетчатый купол, устойчивость, податливость, жёсткость, критическая нагрузка, узел.

ESTIMATION OF THE INFLUENCE OF THE RIGIDITY OF ELASTIC INJECTION OF THE BARS AND INITIAL IMPERFECTIONS OF THE SHAPE ON THE VALUE OF THE CRITICAL NODE LOAD FOR MESH DOMES

\section{Abstract}

The problem of snap through of mesh domes with semi rigid connection of rods at the nodes is considered. The dependences of the change in the critical nodal load on the compliance of the nodes are determined. A condition for the local stability of mesh domes is formulated.

Keywords: mesh dome, stability, pliability, stiffness, critical load, joint.

\section{Введение}

Применение сетчатых куполов обусловлено необходимостью перекрывать большие пролёты с минимальным расходом материалов. Пространственная работа таких конструкций под действием нагрузки, жёсткость, стандартизация элементов, а также эстетические качества являются их преимуществами. Широкому применению сетчатых куполов препятствует малая изученность их работы под нагрузкой и сложность оценки отдельных видов их устойчивости.

Существуют немногочисленные работы, посвящённые вопросу определения напряжённо-деформированного состояния пространственных сетчатых конструкций, а в частности вопросу местной устойчивости сетчатых куполов, в которых комплексно учтено влияние деформаций узловых элементов на величины критических нагрузок [11-16] Во всех перечисленных работах при учёте деформаций изгиба узловых элементов производится пересчёт (редуцирование) момента инерции в плоскости изгиба. Такой подход, безусловно, имеет недостатки [16]. Вместе с тем до настоящего времени практически неисследованным в этом вопросе является влияние начальных несовершенств формы на величину критической узловой нагрузки.

Основная часть. Определение реактивных сил на концах стержней сетчатых куполов и выявление закономерностей деформирования их стержневых многогранников

Целью представленной работы являются оценка влияния жёсткости упругого защемления стержней в узлах и начальных несовершенств формы на величину критической узловой нагрузки, вызывающей продавливание к центру кривизны описанной сфреры отдельных узлов сетчатых куполов.

Процессы деформирования стержневых многогранников в составе сетчатых куполов изначально являются геометрически нелинейными. При этом при решении задачи потери местной устойчивости сетчатых куполов недостаточно рассмотреть отдельно взятый стержневой многогранник без учёта совместного деформирования стержней смежных поясов [2-6] и узловых элементов [12-16].
Для достижения поставленной цели необходимо определиться с достаточной степенью дискретизации системы, определить влияние деформирования стержней смежных поясов на процесс деформирования рассматриваемого стержневого многогранника. В работах [4-6] и в [7-9] было произведено сравнение процессов деформирования стержневых многогранников в составе двухъярусного и трёхъярусного сетчатых куполов как с жёсткими узлами, так и с шарнирными. Был сделан вывод о достаточности рассмотрения духъярусного фрагмента сетчатого купола в силу малости различия ординат диаграмм равновесных состояний: приращение внутренних усилий в стержнях и их вертикальных проекций можно принимать прямо пропорционально изменяющимися относительно перемещений узлов за пределами рассматриваемых двух ярусов. При этом очевидно, что справедливость этого вывода распространяется и на стержневые многогранники сетчатых куполов с упруго-податливыми узлами в силу промежуточности положения в классификации по жёсткости этих узлов относительно названных выше.

На рисунке 1 представлен план радиально-кольцевого сетчатого купола и его фрагмент в пределах смежных плоскостей симметрии.

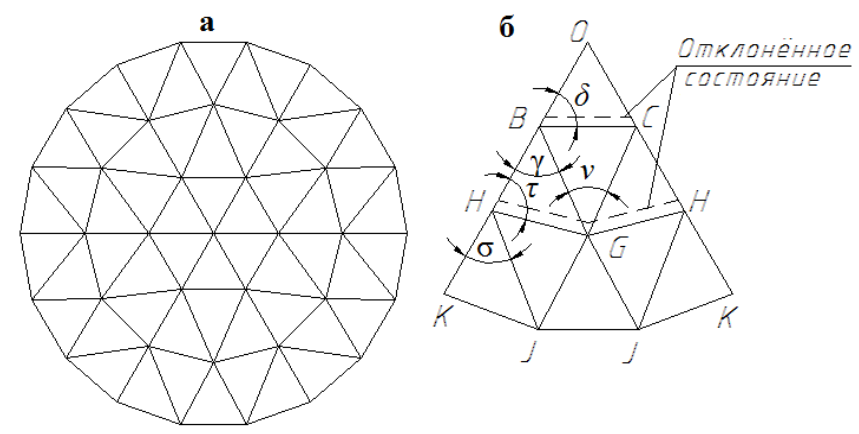

Рисунок 1 - План двухъярусного фрагмента сетчатого купола (а), его сегмент в исходном и в равновесном отклонённом состоянии в плане (б) 
Следует рассмотреть деформирование стержней трёхъярусного фрагмента сетчатого купола, приняв его опирание шарнирноподвижным, однако все стержни опорного контура упруго защемлены в узлах.

Основные предпосылки, принятые в расчёте:

1) форма равновесия симметрична, вертикально действующая внешняя нагрузка, приложенная к узлам трёх- и двухъярусных сетчатых куполов, является осесимметричной;

2) длины стержней различаются незначительно и могут быть приняты одинаковыми;

3) моменты инерции и площади поперечных сечений стержней одинаковы;

4) материал стержней изотропен, деформирование стержней полагается упругим;

5) размерами узлов на напряжённо-дефрормированное состояние сетчатых куполов можно пренебречь, жёсткость упругого защемления стержней в узлах одинакова;

6) воздействия на сетчатые купола являются силовыми;

7) стержни кольцевых рядов горизонтальны как в исходном состоянии, так и в отклонённом равновесном состоянии

Вывод зависимостей, позволяющих определить величины безразмерных параметров продольных деформаций стержней относительно безразмерных параметров перемещений, приведён в работах, посвящённых исследованию местной устойчивости сетчатых куполов с жёсткими узлами $[1,6]$. Принимая неизменным обозначения узлов двухъярусного фрагмента сетчатого купола, эти зависимости можно записать в следующем виде:

$$
\begin{gathered}
\bar{\varepsilon}_{O C}=\frac{N_{O C}}{\rho_{O C}^{2} E A}=\chi_{O C}\left(1-0,5 \chi_{O C}\right)+0,5 \cdot \bar{\varepsilon}_{B C} \sec \delta ; \\
\bar{\varepsilon}_{C H}=\frac{N_{C H}}{\rho_{O C}^{2} E A}=\frac{4 \cos ^{2} \tau}{1+4 \cos ^{2} \tau}\left(9 \chi_{C H} m^{2}\left(1-0,5 \chi_{C H}\right)-0,5 \varepsilon_{B C} \sec \delta\right) ;(2) \\
\quad-N_{C G}=\frac{N_{C G}}{\rho_{O C}^{2} E A}=0,5 \cdot \bar{\varepsilon}_{C H} \sec 0,5 v ; \\
\bar{\varepsilon}_{B C}=\frac{N_{B C}}{\rho_{O C}^{2} E A}=2 \cos \delta \frac{36 m^{2} \chi_{C H}\left(1-0,5 \chi_{C H}\right) \cos ^{2} \tau(1+\cos \gamma \cdot \sec 0,5 v)}{\left(1+4 \cos ^{2} \tau\right)\left(1+4 \cos ^{2} \delta\right)+4 \cos ^{2} \tau(1+\cos \gamma \cdot \sec 0,5 v)}- \\
-2 \cos \delta \frac{\chi_{O C}\left(1-0,5 \chi_{O C}\right)\left(1+4 \cos ^{2} \tau\right)}{\left(1+4 \cos ^{2} \tau\right)\left(1+4 \cos ^{2} \delta\right)+4 \cos ^{2} \tau(1+\cos \gamma \cdot \sec 0,5 v)},
\end{gathered}
$$

где $\bar{\varepsilon}$ - безразмерный параметр продольной деформации стержня;

$\rho_{O C}$ - угол наклона стержней типа ОС к касательной плоскости, проведённой к описанной сфере, рад;

$X_{i j}$ - безразмерный параметр перемещения узла $і$ относительно узла $j$, отношение угла поворота хорды стержня относительно начального положения к начальному углу её наклона $\rho_{i j}$ (если описанная поверхность - сфера, то $\left.\rho_{C H}=3 \rho_{O C}\right)$;

$N_{i j}$ - распор на концах стержня, численно равный по абсолютной величине продольной силе, $\mathrm{H}$;

$m$ - коэффициент, учитывающий отклонения от соотношений величин начальных углов наклона стержней от 1/3: при его значениях больших единицы форма верхнего стержневого многогранника более пологая, чем необходимо, в противном случае - более выпуклая [1].

В работах [1-3] изложено построение методики проверки устойчивости сетчатых куполов с жёсткими узлами, в которых было учтено влияние продольно-поперечного изгиба в стержнях и наличие начальных несовершенств. В полученном решении использованы функции продольного изгиба, которые входят в зависимости для определения изгибающих моментов и поперечных сил на концах стержней, однако они не учитывают деформации узловых элементов на величины этих внутренних усилий. Для установления совместного влияния продольно-поперечного изгиба и деформирования узлового элемента на величины изгибающих моментов и поперечных сил на концах стержней следует использовать дифференциальное уравнение продольного изгиба:

$$
E l \frac{d^{4} w}{d x^{4}}+N \frac{d^{2} w}{d x^{2}}=0
$$

общее решение которого запишется в следующем виде:

$$
w=C_{1} \sin \left(u \cdot \frac{x}{L}\right)+C_{2} \cos \left(u \cdot \frac{x}{L}\right)+C_{3} \cdot x+C_{4},
$$

где $L$ - длина стержня, м,

$U$ - параметр продольной силы в стержне:

$$
u=I \sqrt{\frac{N}{E l}}=\sqrt{\frac{\overline{\varepsilon \rho_{O C}^{2} L^{2} E A}}{E l}}=n \sqrt{\bar{\varepsilon}},
$$

$n$ - эквивалентная гибкость, параметр, связывающий геометрические характеристики подобранного сечения и геометрические характеристики стержневого многогранника:

$$
n=\sqrt{\frac{L^{2} \rho_{O C}^{2} E A}{E l}}=\frac{\rho_{O C} \cdot L}{i}=\frac{\Delta_{1}}{i},
$$

$\Delta_{1}$ - стрела подъёма стержневого многогранника, м,

$i$ - радиус инерции поперечного сечения, м.

Поскольку стержни сетчатых куполов в рассматриваемой задаче упруго защемлены в узлах, необходимо определить зависимости изменения изгибающих моментов и поперечных сил на концах стержней при двух схемах десормирования: смещение одной из заделок и её поворот. На рисунке 2 показаны рассматриваемые схемы деформирования произвольного стержня.

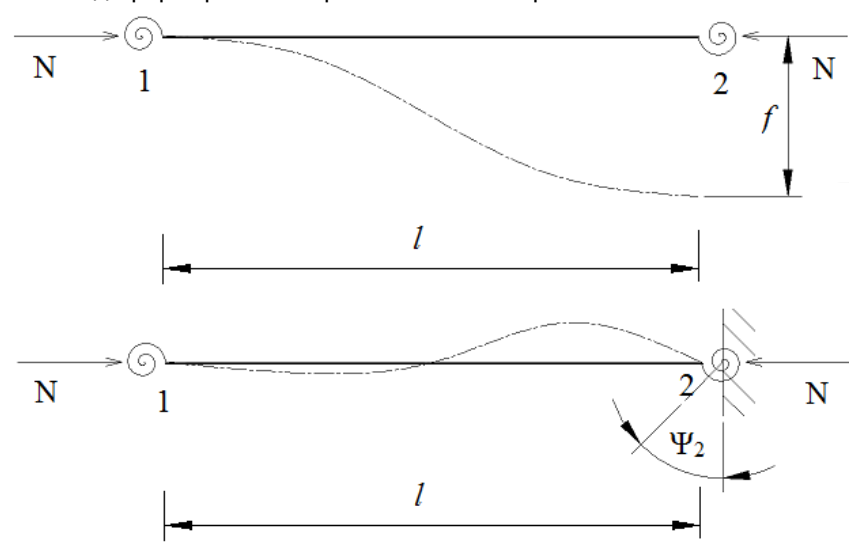

Рисунок 2 - Рассматриваемые варианты деформирования стержня

Граничные условия для уравнения (6) записаны в таблице 1.

Таблица 1 - Граничные условия для различных вариантов десормирования стержня

\begin{tabular}{|c|c|}
\hline Смещение заделки & \multicolumn{1}{|c|}{ Поворот заделки } \\
\hline $\begin{array}{l}w(0)=0 ; \\
w(L)=f ; \\
w^{\prime}(0)=\frac{E l}{R} w^{\prime \prime}(0) ; \\
w^{\prime}(L)=-\frac{E l}{R} w^{\prime \prime}(L),\end{array}$ & $\left\{\begin{array}{l}w(0)=0 ; \\
w(L)=0 ; \\
w^{\prime}(0)=\frac{E l}{R} w^{\prime \prime}(0) ; \\
w^{\prime}(L)+\Psi_{2}=-\frac{E l}{R} w^{\prime \prime}(L),\end{array}\right.$ \\
\hline
\end{tabular}

В приведенных в таблице уравнениях $f$ - величина смещения упругой заделки, м, $\Psi_{2}$ - угол поворота второй упругой заделки, рад, $E I$ - изгибная жёсткость стержня в плоскости изгиба, $\mathrm{HM}^{2}, R$ - жёсткость упругого защемления стержня в узловом элементе, Нм/рад.

Опуская промежуточные вычисления, связанные с определением значение коэфффициентов $C_{1}-C_{4}$ для обоих случаев, можно записать выражения для вычисления изгибающих моментов и реактивных сил на концах: 
1) смещение упругой заделки на величину $f$

$$
\begin{aligned}
& M_{1}=M_{2}=6 \frac{E l}{L^{2}} f \cdot \Phi_{4}(u, r) ; \\
& V_{1}=V_{2}=12 \frac{E l}{L^{3}} f \cdot \mathrm{H}_{2}(u, r) ;
\end{aligned}
$$

2) поворот упругой заделки на величину $\Psi_{2}$ :

$$
\begin{aligned}
& M_{1}=2 \frac{E l}{L} \Psi_{2} \cdot \Phi_{3}(u, r) ; \\
& M_{2}=4 \frac{E l}{L} \Psi_{2} \cdot \Phi_{2}(u, r) ; \\
& V_{1}=V_{2}=12 \frac{E l}{L^{2}} \Psi_{2} \cdot \Phi_{4}(u, r),
\end{aligned}
$$

где функции $\Phi_{2}(u, r), \Phi_{3}(u, r), \Phi_{4}(u, r), \mathrm{H}_{2}(u, r)$ зависят от безразмерного параметра продольной силы и безразмерного параметра жёсткости $r$.

$$
\begin{gathered}
\Phi_{2}(u, r)=\frac{r u\left(\sin u\left(r+u^{2}\right)-r u \cdot \cos u\right)}{4\left(\left(2 r-r^{2}+u^{2}\right) u \sin u-\left(r+u^{2}\right) 2 r \cdot \cos u+2 r^{2}\right)} \\
\Phi_{3}(u, r)=\frac{r^{2} u(u-\sin u)}{2\left(\left(2 r-r^{2}+u^{2}\right) u \sin u-\left(r+u^{2}\right) 2 r \cdot \cos u+2 r^{2}\right)} \\
\Phi_{4}(u, r)=\frac{r u^{2}(r \cdot(1-\cos u)+u \sin u)}{6\left(\left(2 r-r^{2}+u^{2}\right) u \sin u-\left(r+u^{2}\right) 2 r \cdot \cos u+2 r^{2}\right)} \\
\mathrm{H}_{2}(u, r)=\frac{r u^{3}(u \cdot(1+\cos u)+r \sin u)}{12\left(\left(2 r-r^{2}+u^{2}\right) u \sin u-\left(r+u^{2}\right) 2 r \cdot \cos u+2 r^{2}\right)}
\end{gathered}
$$

где $r$ - безразмерный параметр жёсткости:

$$
r=\frac{R L}{E l} .
$$

При устремлении к бесконечности величины безразмерного параметра жёсткости значения функций (15)-(18) в пределе будут равны значениям одноимённых функций устойчивости [10]:

$$
\begin{aligned}
& \lim _{r \rightarrow \infty} \Phi_{2}(u, r)=\varphi_{2}(u)=\frac{u(\operatorname{tg} u-u)}{8 \operatorname{tg} u \cdot\left(\operatorname{tg} \frac{u}{2}-\frac{u}{2}\right)} \\
& \lim _{r \rightarrow \infty} \Phi_{3}(u, r)=\varphi_{3}(u)=\frac{u \cdot(u-\sin u)}{4 \sin u \cdot\left(\operatorname{tg} \frac{u}{2}-\frac{u}{2}\right)} ; \\
& \lim _{r \rightarrow \infty} \Phi_{4}(u, r)=\varphi_{4}(u)=\frac{u^{2} \operatorname{tg} \frac{u}{2}}{12 \cdot\left(\operatorname{tg} \frac{u}{2}-\frac{u}{2}\right)} ; \\
& \lim _{r \rightarrow \infty} \mathrm{H}_{2}(u, r)=\eta_{2}(u)=\frac{u^{3}}{24 \cdot\left(\operatorname{tg} \frac{u}{2}-\frac{u}{2}\right)} .
\end{aligned}
$$

Графики представленных функций (15)-(18) приведены на рисунке 3 для некоторых величин параметров продольных сил.

Из представленных графиков видно, что ординаты функций $\Phi_{2}(u, r), \Phi_{3}(u, r), \Phi_{4}(u, r), H_{2}(u, r)$ изменяются не по линейной зависимости от безразмерного параметра жёсткости узлов $r$, в то время как зависимость изменения величины коэфффициента редуцирования момента инерции поперечного сечения стержней, представленная в работе [16], имеет линейный вид. Графики представленных функций скорее можно интерпретировать как билинейные, при этом методика определения величин критических узловых нагрузок, представленная в работе [16], будет иметь приемлемую точность при параметре $r \approx 300$ и более, а также при достаточно больших величинах квадрата эквивалентной гибкости $n^{2} \geq 40$, когда величины поперечных сил в стержнях в уравнении равновесия между вертикальными проекциями внутренних усилий и внешней узловой нагрузкой мало по сравнению с вертикальными проекциями продольных сил [1-6].
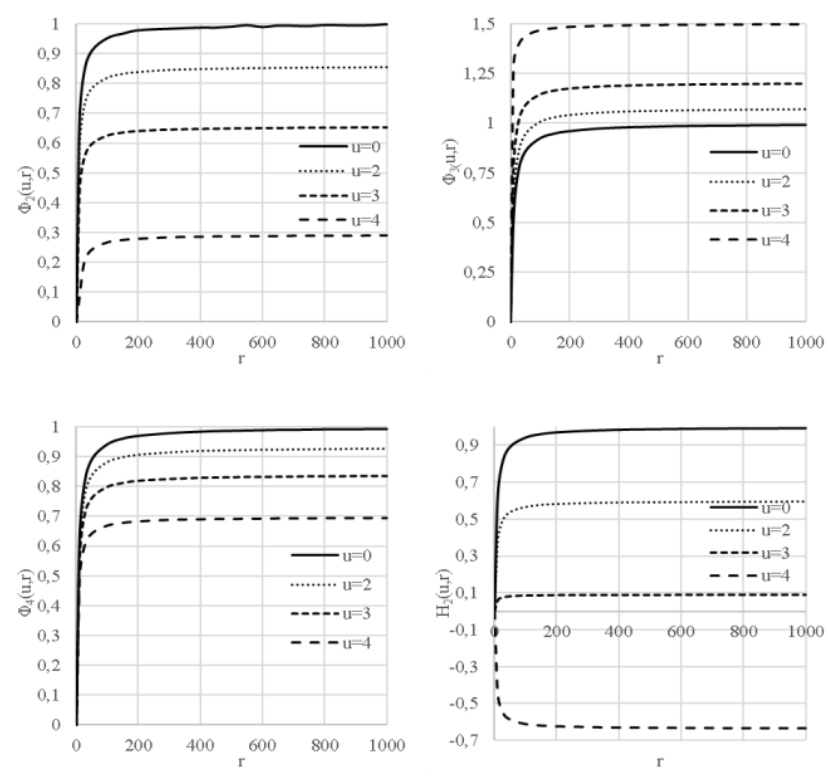

Рисунок 3 - Графики изменения функций $\Phi_{2}(u, r), \Phi_{3}(u, r), \Phi_{4}(u, r), \mathrm{H}_{2}(u, r)$

При отсутствии сосредоточенных моментов одно из уравнений равновесия узла $C$ можно записать в следующем виде:

$$
\Sigma M_{C}^{C H}+2 \cos \gamma \cdot \Sigma M_{C}^{C G}=\Sigma M_{C}^{O C}+2 \cos \delta \cdot \Sigma M_{C}^{B C},
$$

где $\Sigma M_{C}^{C H}$ - величина изгибающего момента в стержне типа $\mathrm{CH}$ в узле $C$, Нм, остальные записи в формуле расшифрровываются аналогично; следует отметить, что величины изгибающих моментов составлены с учётом зависимостей (15)-(18) аналогично полученным выражениям в работе [5].

Из уравнения (24) можно получить выражение для определения параметра угла поворота узла $C, H$ и $G$ в зависимости от параметров перемещений концов стержней $X_{i j}$ :

$$
\Theta_{C}=\frac{\theta_{C}}{\rho_{O C}}=\frac{F\left(\chi_{O C}, \chi_{C H}\right)}{\Phi(\chi, \eta)},
$$

где функции $F(X, \eta)$ и $\Phi(X, \eta)$ могут быть записаны в следующем виде:

$$
\begin{gathered}
F\left(\chi_{O C}, \chi_{C H}\right)=3 \chi_{O C} \Phi_{4}\left(u_{O C}\right)+9 \chi_{C H} m\left(\Phi_{4}\left(u_{C H}\right)+2 \Phi_{4}\left(u_{C G}\right) \cos \gamma\right)- \\
-\eta m\left(B_{H} \Phi_{3}\left(u_{C H}\right)+2 B_{G} \Phi_{3}\left(u_{C G}\right) \cos \gamma \cos 0,5 v\right) ; \\
\Phi\left(\chi_{O c}, \chi_{C H}\right)=2\left(\Phi_{2}\left(u_{C H}\right)+\Phi_{2}\left(u_{O C}\right)\right)+A_{H} \Phi_{3}\left(u_{C H}\right)+2 \cos ^{2} \delta\left(2 \Phi_{2}\left(u_{B C}\right)-\Phi_{3}\left(u_{B C}\right)\right)+ \\
+2 \cos \gamma\left(2 \Phi_{2}\left(u_{C G}\right) \cos \gamma+A_{G} \Phi_{3}\left(u_{C G}\right) \cos 0,5 v\right),
\end{gathered}
$$

где, в свою очередь,

$$
A_{H}=-\frac{\cos ^{2} \tau\left(\Phi_{3}\left(u_{C G}\right) \cos \gamma \cos 0,5 v+\Phi_{3}\left(u_{C H}\right)\right)+\Phi_{2}\left(u_{C G}\right) \Phi_{3}\left(u_{C H}\right) \cos ^{2} 0,5 v}{Z} ;
$$

$B_{H}=m \cdot 9 \frac{\cos ^{2} \tau\left(\Phi_{4}\left(u_{C G}\right) \cos 0,5 v+\Phi_{4}\left(u_{C H}\right)\right)+\Phi_{2}\left(u_{C G}\right) \Phi_{4}\left(u_{C H}\right) \cos ^{2} 0,5 v}{Z}$

$A_{G}=-\frac{\cos ^{2} \tau\left(4 \Phi_{3}\left(u_{C G}\right) \cos \gamma \cdot \cos 0,5 v+\Phi_{3}\left(u_{C H}\right)\right)}{2 Z}-\frac{2 \Phi_{2}\left(u_{C H}\right) \Phi_{3}\left(u_{C G}\right) \cos 0,5 v \cdot \cos \gamma}{2 Z}$

$B_{G}=m \cdot 9 \frac{\cos ^{2} \tau\left(4 \Phi_{4}\left(u_{C G}\right) \cos 0,5 v+\Phi_{4}\left(u_{C H}\right)\right)+2 \Phi_{2}\left(u_{C H}\right) \Phi_{4}\left(u_{C G}\right) \cos 0,5 v}{2 Z}$,

где знаменатель $Z$ определяется из следующего выражения: 


$$
\begin{gathered}
Z=\cos ^{2} \tau\left(4 \Phi_{2}\left(u_{C G}\right) \cos ^{2} 0,5 v+2 \Phi_{2}\left(u_{C H}\right)+3 \cos ^{2} \tau\right)+ \\
+2 \Phi_{2}\left(u_{C G}\right) \Phi_{2}\left(u_{C H}\right) \cos ^{2} 0,5 v \\
\Theta_{H}=\frac{\theta_{H}}{\rho_{O C}}=A_{H} \Theta_{C}+\chi_{C H} \cdot B_{H} \\
\Theta_{G}=\frac{\theta_{G}}{\rho_{O C}}=A_{G} \Theta_{C}+\chi_{C H} \cdot B_{G}
\end{gathered}
$$

Величины безразмерных параметров реактивных сил с учётом изложенных выше зависимостей можно выразить в следующем виде:

$$
\begin{gathered}
\bar{V}^{O C}=\frac{V^{O C}}{\rho_{O C}^{3} E A}=-12 \frac{\chi_{O C}}{n^{2}} \mathrm{H}_{2}\left(u_{O C}, r\right)+6 \frac{\Theta_{C}}{n^{2}} \Phi_{4}\left(u_{O C}, r\right) ;(35) \\
\bar{V}^{B C}=\frac{V^{B C}}{\rho_{O C}^{3} E A}=0 ; \\
\bar{V}^{C H}=\frac{V^{C H}}{\rho_{O C}^{3} E A}=-36 m \frac{\chi_{C H}}{n^{2}} \mathrm{H}_{2}\left(u_{C H}, r\right)+\frac{6}{n^{2}}\left(\Theta_{C}+\Theta_{H}\right) \Phi_{4}\left(u_{C H}, r\right) ;(37) \\
\bar{V}^{C G}=\frac{V^{C G}}{\rho_{O C}^{3} E A}=-36 m \frac{\chi_{C H}}{n^{2}} H_{2}\left(u_{C G}, r\right)+\frac{6}{n^{2}}\left(\Theta_{C} \cos \gamma+\Theta_{G} \cos 0,5 v\right) \Phi_{4}\left(u_{C G}, r\right) .
\end{gathered}
$$

Уравнения равновесия внешних и внутренних сил, сосредоточенных в узлах, можно соотнести:

$$
\frac{P_{C}}{P_{O}}=\frac{\left(N_{C H}+2 N_{C G}\right) \cdot 3 m \rho_{O C}\left(1-\chi_{C H}\right)-N_{O C} \rho_{O C}\left(1-\chi_{O C}\right)+V_{O C}-V_{C H}-2 V_{C G}}{k\left(N_{O C} \rho_{O C}\left(1-\chi_{O C}\right)-V_{O C}\right)} .
$$

Разделив в правой части равенства числитель и знаменатель на $\rho_{O C}^{3} E A$, можно получить следующее уравнение, которое решается относительно безразмерных параметров перемещений узлов $\chi_{\text {сH }}$ нижнего яруса рассматриваемого двухъярусного фрагмента сетчатого купола, при задании параметров перемещения узлов верхнего яруса $\chi_{\text {ос }}$ и эквивалентной гибкости $n$ и безразмерного параметра жёсткости $r$.

$$
\Omega=\frac{P_{C}}{P_{O}}=\frac{3 m\left(\bar{\varepsilon}_{C H}+2 \bar{\varepsilon}_{C G}\right)\left(1-\chi_{C H}\right)-\bar{\varepsilon}_{O C}\left(1-\chi_{O C}\right)+\bar{V}_{O C}-\bar{V}_{C H}-2 \bar{V}_{C G}}{k\left(\bar{\varepsilon}_{O C}\left(1-\chi_{O C}\right)-\bar{V}_{O C}\right)}
$$

График сопротивления стержней многогранника сосредоточенной нагрузке, приложенной к его верхнему узлу, описывается зависимостью, приведённой в знаменателе правой части выражения (40):

$$
p=k\left(\bar{\varepsilon}_{O C}\left(1-\chi_{O C}\right)-\bar{V}_{O C}\right),
$$

где $p_{0}$ - безразмерный параметр нагрузки в узле $O$.

При подстановке величин $\chi_{O c}, \chi_{C H}$ в уравнение (41) при фиксировании переменных $r, n, m$ можно построить графические зависимости изменения приращений вертикальных проекций внутренних усилий в стержнях вблизи узла $O$, которые в каждом случае будут являться диаграммами равновесных состояний. На рисунке 4 изображены диаграммы равновесных состояний для шестиугольного и пятиугольного в плане стержневых многогранников, полученные для параметра эквивалентной гибкости $n=1$, коэфффициенте, учитывающем несовершенства формы $m=1$ (отклонения от проектного положения отсутствуют), и различных безразмерных параметров податливостей $r$ и неравномерности осесимметричного нагружения $\Omega=1$ узловой нагрузкой.

На диаграммах равновесных состояний, изображённых на рисунке 4, на выносках обозначены величины критических параметров узловых нагрузок. При превышении этих значений равновесие между внешней нагрузкой вертикальными проекциями внутренних усилий в стержнях стержневых многогранников невозможно в их выпуклом состоянии и достигается только в вогнутом. Условие устойчивости сетчатого купола в случае продавливания его узлов можно сформулировать следующим образом:

$$
\frac{P_{y 3} L^{3}}{\Delta_{1}^{3} E A}<p_{\kappa p}
$$

где величина $p_{\kappa p}$ - значение критического безразмерного параметра нагрузки;

$P_{\text {уз }}$ - узловая нагрузка, приложенная к центральному узлу рассматриваемого стержневого многогранника, $\mathrm{H}$.
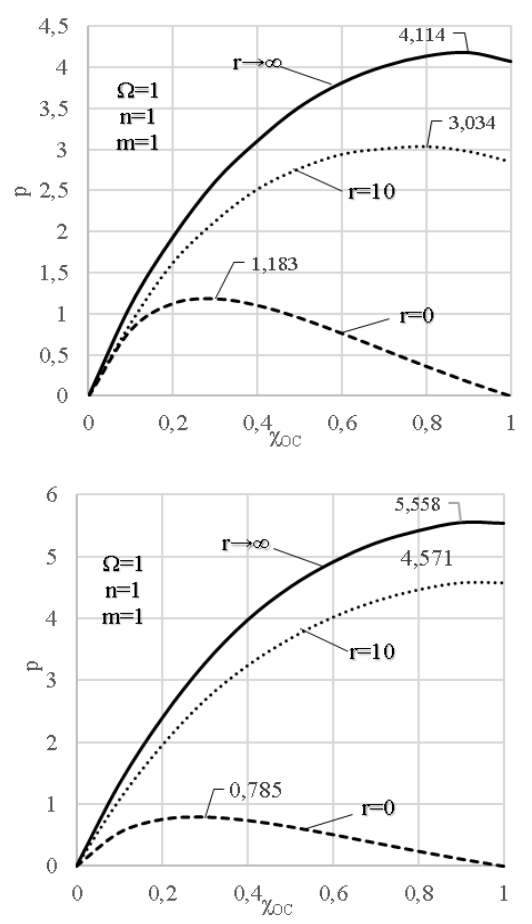

Рисунок 4 - Диаграммы равновесных состояний шестиугольного (а) и пятиугольного (б) в плане стержневых многогранников при $r=0, r=10, r \rightarrow \infty$

Варьируя параметры $\Omega, n, m, r$, можно получить серии подобных диаграмм, представленным на рисунке 3. Были вычислены значения критических параметров нагрузок. При этом, обратившись к уравнениям (15)-(18) и (35) (38), можно сделать вывод, что устремление к бесконечности параметра $n$ и к нулю параметра $r$ в равной степени приводит в пределе к равенству нулю величин поперечных сил в стержнях. В этом случае характер деформирования сетчатого купола с упруго-податливыми узлами стремится к шарнирной модели. Определение величин критических нагрузок, вызывающих продавливание к центру кривизны описанной сферы узлов шарнирно-стержневых сетчатых куполов, представлено в работе [4]. Безразмерные параметры критических узловых нагрузок, полученные для шарнирностержневых сетчатых куполов приведены в таблице 2.

Таблица 2 - Параметры критических узловых нагрузок, полученные для шарнирно-стержневых сетчатых куполов

\begin{tabular}{|c|c|c|c|c|}
\hline \multirow{2}{*}{$\begin{array}{c}\text { Число граней } \\
\text { многогранника }\end{array}$} & \multicolumn{4}{|c|}{$p$} \\
\hline 6 & 1.183 & $\Omega=0,5$ & $\Omega=0,25$ & $\Omega=0$ \\
\hline 5 & 0.785 & 0.823 & 0.722 & 0.620 \\
\hline
\end{tabular}

Взяв за основу безразмерные параметры критических узловых нагрузок, полученные для шарнирно-стержневых сетчатых куполов, можно соотнести с ними полученные величины критических узловых нагрузок при аналогичных остальных параметрах. Результаты отношения величин безразмерных параметров критических нагрузок, вызывающих продавливание к центру кривизны описанной сферы узлов сетчатых куполов с упруго-податливыми и шарнирными узлами, изложены в таблице 3. 
Вестник Брестского государственного технического университета. 2021 Таблица 3 - Коэффициенты соотношений между величинами параметров критических нагрузок в сетчатых куполах с упруго-податливыми и шарнирными узлами

\begin{tabular}{|c|c|c|c|c|c|c|c|c|c|c|c|c|c|c|}
\hline \multirow{3}{*}{$\begin{array}{c}\text { Число граней } \\
\text { многогранника }\end{array}$} & \multirow{3}{*}{$r$} & \multirow{3}{*}{$n^{2}$} & \multicolumn{12}{|c|}{$\Omega$} \\
\hline & & & \multicolumn{4}{|c|}{$m=0.8$} & \multicolumn{4}{|c|}{$m=1$} & \multicolumn{4}{|c|}{$m=1.2$} \\
\hline & & & 1 & 0.5 & 0.25 & 0 & 1 & 0.5 & 0.25 & 0 & 1 & 0.5 & 0.25 & 0 \\
\hline 1 & 2 & 3 & 4 & 5 & 6 & 7 & 8 & 9 & 10 & 11 & 12 & 13 & 14 & 15 \\
\hline \multirow{24}{*}{6} & \multirow{8}{*}{8} & 1 & 3.255 & 6.752 & - & - & 3.478 & - & - & - & 4.117 & - & - & - \\
\hline & & 1.5 & 2.334 & 5.043 & 8.681 & - & 2.669 & 6.147 & - & - & 3.3 & - & - & - \\
\hline & & 2 & 1.413 & 3.334 & 7.132 & 11.88 & 1.860 & 4.383 & 7.351 & - & 2.482 & - & - & - \\
\hline & & 3 & 1.018 & 2.425 & 4.036 & 8.739 & 1.860 & 3.425 & 5.612 & - & 2.032 & 4.666 & - & - \\
\hline & & 5 & 0.761 & 1.795 & 2.937 & 5.99 & 1.445 & 2.649 & 4.211 & 6.039 & 1.636 & 3.321 & 5.098 & - \\
\hline & & 10 & 0.603 & 1.378 & 2.082 & 3.311 & 1.170 & 1.847 & 1.641 & 3.694 & 1.185 & 2.141 & 2.819 & 3.966 \\
\hline & & 20 & 0.719 & 1.103 & 1.358 & 1.782 & 0.934 & 1.228 & 1.476 & 1.834 & 0.878 & 1.286 & 1.529 & 1.856 \\
\hline & & 40 & 0.648 & 0.919 & 0.965 & 1.106 & 0.818 & 0.872 & 0.951 & 1.106 & 0.714 & 0.887 & 0.969 & 1.102 \\
\hline & \multirow{8}{*}{ ి్లి } & 1 & 3.093 & 6.415 & - & - & 3.419 & 6.258 & - & - & 3.912 & - & - & - \\
\hline & & 1.5 & 2.218 & 4.791 & 8.247 & - & 2.489 & 5.840 & - & - & 3.135 & - & - & - \\
\hline & & 2 & 1.343 & 3.167 & 6.776 & - & 1.640 & 3.944 & 7.131 & - & 2.358 & - & - & - \\
\hline & & 3 & 0.967 & 2.304 & 3.834 & 8.302 & 1.452 & 3.395 & 5.652 & - & 1.931 & 4.433 & - & - \\
\hline & & 5 & 0.723 & 1.705 & 2.79 & 5.691 & 1.178 & 2.659 & 4.209 & 6.032 & 1.554 & 3.155 & 4.843 & - \\
\hline & & 10 & 0.573 & 1.309 & 1.978 & 3.146 & 0.944 & 1.838 & 2.498 & 3.597 & 1.126 & 2.034 & 2.678 & 3.768 \\
\hline & & 20 & 0.683 & 1.048 & 1.29 & 1.693 & 0.866 & 1.228 & 1.486 & 1.823 & 0.834 & 1.221 & 1.452 & 1.764 \\
\hline & & 40 & 0.615 & 0.873 & 0.916 & 1.051 & 0.799 & 0.878 & 0.972 & 1.100 & 0.679 & 0.843 & 0.92 & 1.047 \\
\hline & \multirow{8}{*}{ 으 } & 1 & 2.469 & 5.79 & - & - & 2.565 & 6.015 & 10.13 & - & 3.684 & - & - & - \\
\hline & & 1.5 & 2.285 & 5.356 & 0.48 & - & 2.252 & 5.272 & 8.836 & - & 3.281 & - & - & - \\
\hline & & 2 & 1.732 & 4.057 & 1.921 & 9.22 & 1.939 & 4.530 & 7.545 & - & 2.879 & - & - & - \\
\hline & & 3 & 0.994 & 2.323 & 3.842 & 7.894 & 1.314 & 3.046 & 4.963 & - & 2.074 & - & - & - \\
\hline & & 5 & 0.804 & 1.847 & 2.923 & 5.24 & 1.123 & 2.461 & 3.698 & 5.357 & 1.631 & 3.224 & - & - \\
\hline & & 10 & 0.713 & 1.445 & 1.939 & 2.673 & 0.986 & 1.702 & 2.140 & 2.811 & 1.179 & 1.863 & 2.274 & 2.895 \\
\hline & & 20 & 0.821 & 1.182 & 1.317 & 1.556 & 0.952 & 1.194 & 1.347 & 1.563 & 0.926 & 1.203 & 1.357 & 1.563 \\
\hline & & 40 & 0.786 & 1.03 & 1.001 & 1.069 & 0.930 & 0.931 & 0.974 & 1.056 & 0.802 & 0.899 & 0.956 & 1.045 \\
\hline \multirow{24}{*}{5} & \multirow{8}{*}{8} & 1 & 7.169 & - & - & - & 7.628 & - & - & - & - & - & - & - \\
\hline & & 1.5 & 4.993 & 10.02 & - & - & 5.638 & 12.78 & - & - & - & - & - & - \\
\hline & & 2 & 2.817 & 8.383 & 10.3 & - & 3.648 & 9.378 & - & - & - & - & - & - \\
\hline & & 3 & 1.989 & 5.103 & 8.12 & - & 2.808 & 7.123 & - & - & 3.98 & - & - & - \\
\hline & & 5 & 1.466 & 3.682 & 5.697 & 8.393 & 2.158 & 5.193 & 7.118 & - & 2.764 & 5.645 & - & - \\
\hline & & 10 & 1.12 & 2.542 & 3.411 & 4.612 & 1.532 & 3.049 & 3.852 & 5.042 & 1.79 & 3.343 & 4.122 & 5.636 \\
\hline & & 20 & 0.962 & 1.731 & 2.037 & 2.48 & 1.107 & 1.821 & 2.127 & 2.522 & 1.158 & 1.885 & 2.173 & 2.544 \\
\hline & & 40 & 0.898 & 1.255 & 1.162 & 1.575 & 0.736 & 1.261 & 1.395 & 1.570 & 0.827 & 1.259 & 1.393 & 1.565 \\
\hline & \multirow{8}{*}{ ষ্ల } & 1 & 6.596 & - & - & - & 7.018 & - & - & - & - & - & - & - \\
\hline & & 1.5 & 4.594 & 9.221 & - & - & 5.187 & 11.75 & - & - & - & - & - & - \\
\hline & & 2 & 2.591 & 7.712 & 9.474 & - & 3.357 & 8.628 & - & - & - & - & - & - \\
\hline & & 3 & 1.829 & 4.694 & 7.47 & - & 2.583 & 6.553 & - & - & 3.661 & - & - & - \\
\hline & & 5 & 1.349 & 3.387 & 5.242 & 7.722 & 1.985 & 4.778 & 6.548 & - & 2.543 & 5.193 & - & - \\
\hline & & 10 & 1.03 & 2.339 & 3.138 & 4.243 & 1.410 & 2.805 & 3.544 & 4.639 & 1.647 & 3.075 & 3.793 & 5.185 \\
\hline & & 20 & 0.885 & 1.593 & 1.874 & 2.282 & 1.018 & 1.676 & 1.957 & 2.321 & 1.065 & 1.734 & 1.999 & 2.34 \\
\hline & & 40 & 0.826 & 1.154 & 1.069 & 1.449 & 0.677 & 1.160 & 1.283 & 1.444 & 0.761 & 1.158 & 1.281 & 1.439 \\
\hline & \multirow{8}{*}{ 으 } & 1 & 4.926 & - & - & - & 5.911 & - & - & - & - & - & - & - \\
\hline & & 1.5 & 4.172 & - & - & - & 5.126 & - & - & - & - & - & - & - \\
\hline & & 2 & 3.418 & - & - & - & 4.341 & - & - & - & - & - & - & - \\
\hline & & 3 & 1.91 & 4.805 & 7.503 & - & 2.772 & 6.764 & - & - & 3.746 & - & - & - \\
\hline & & 5 & 1.504 & 3.593 & 5.141 & - & 2.130 & 4.626 & - & - & 2.609 & - & - & - \\
\hline & & 10 & 1.22 & 2.394 & 2.947 & 3.704 & 1.492 & 2.643 & 3.125 & 3.821 & 1.611 & 2.768 & 3.240 & 3.884 \\
\hline & & 20 & 1.164 & 1.704 & 1.912 & 2.195 & 1.140 & 1.727 & 1.933 & 2.201 & 1.129 & 1.739 & 1.938 & 2.198 \\
\hline & & 40 & 1.056 & 1.361 & 1.436 & 1.557 & 0.934 & 1.318 & 1.411 & 1.541 & 0.883 & 1.292 & 1.393 & 1.530 \\
\hline
\end{tabular}


Проверку устойчивости формы сетчатых куполов, деформации изгиба узлов, которые не могут быть приняты малыми, необходимо производить в следующей последовательности:

1) на основе исходных данных определить площадь поперечного сечения стержней $A$, радиус инерции стержней в радиальных плоскостях $i$, стрелу подъёма $\Delta_{1}$ исследуемого стержневого многогранника, величины узловых нагрузок в его вершине $P_{0}$ и в окружающих его узлах $P_{C}$, рассчитанных в результате сбора нагрузок на покрытие; загружение области, для узлов которой вычисляются критические параметры нагрузок, в этом случае не должно значительно отличаться от осесимметричного в пределах двух смежных ярусов;

2) определить на основе исходных данных величину эквивалентной гибкости $n$ стержневого многогранника по формуле (8), неравномерность осесимметричного загружения $\Omega$, при проверке устойчивости формы существующей конструкции, форма которой известна, необходимо определить также коэфффициент $m$, учитывающий начальное геометрическое несовершенство формы стержневого многогранника, в случае проверки устойчивости формы проектируемой конструкции $m=1$;

3) выбранную величину критического параметра нагрузки из таблицы 2 , соответствующую форме стержневого многогранника и неравномерности осесимметричного загружения $\Omega$, необходимо умножить на величину соответствующего коэфффициента, взятого в таблице 3 ; в случае промежуточных значений безразмерных параметров $r$ и $\Omega$ следует воспользоваться линейной интерполяцией;

4) результат произведения необходимо сравнить с параметром приложенной нагрузки, используя неравенство (42).

Знак «-» в таблице 3 означает, что при заданных параметрах $r, n, \Omega, m$ диаграмма равновесных состояний стержневого многогранника не имеет точек бифуркации, является монотонно возрастающей. В ряде случаев величины критических параметров нагрузок в сетчатых куполах с упруго-податливыми либо с жёсткими узлами меньше, чем аналогичные значения в сетчатых куполах, узлы которых являются шарнирными. Это обусловлено тем, что величины реактивных сил на концах стержней, появляющиеся вследствие изгиба и входящие в уравнения равновесия (39), направлены в сторону действия внешней нагрузки. Это явление проявляется вследствие учёта продольно-поперечного изгиба, поскольку эпюра изгибающих моментов в этом случае имеет криволинейное очертание и немонотонное изменение ординат, при этом максимальные значения изгибающих моментов находятся не на концах стержня, а вблизи их. На рисунке 5 показаны примеры очертания эпюр изгибающих моментов в стержне при смещении и повороте одной из его упругих заделок при разных параметрах продольной силы и и при принятых величинах $E I=1, L=1, f=1, \Psi_{2}=0$.

Было произведено сравнение диаграмм равновесных состояний, полученных аналитически по предложенной методике, и диаграмм деформирования для верхнего стержневого многогранника шарнирно опёртых двухъярусных сетчатых куполов, сеть которых построена по радиально-кольцевой схеме (рисунок 1). Выбранный тип сечения труба, внешний диаметр которой 60 мм, толщина стенки 4 мм. Длины стержней меридионального направления - 1250 мм, стрела подъёма верхнего яруса 20 мм $\left(n^{2}=1\right)$ и 45 мм $\left(n^{2}=5\right)$, форма стержневого многогранника в плане - правильный шестиугольник. Опирание рассматриваемого двухъярусного фрагмента сетчатого купола принималось шарнирно-подвижным. На рисунке ба показан рассмотренный способ приложения перемещений: ко всем узлам, на рисунке 6(б) размеры двухъярусного фрагмента сетчатого купола в плане, на рисунке 6(в) - общий вид расчётной модели. Перемещения найдены аналитически по описанной выше методике так, чтобы суммы вертикальных проекций внутренних усилий были равны $(\Omega=1)$. Такое сравнение результатов, безусловно, является выборочным из произвольного количества вариантов, однако не влияет на общность полученных выводов.

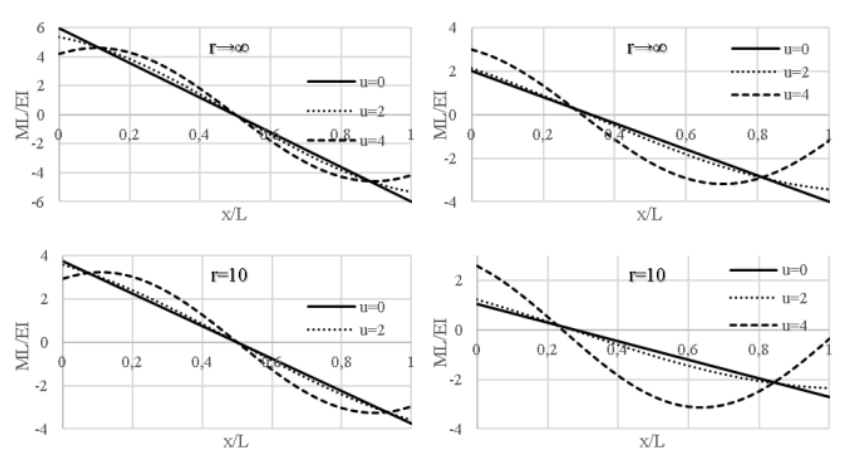

Рисунок 5 - Формы безразмерных эпюр изгибающих моментов при единичном смещении или повороте одной из упругих заделок и различных величинах $r$

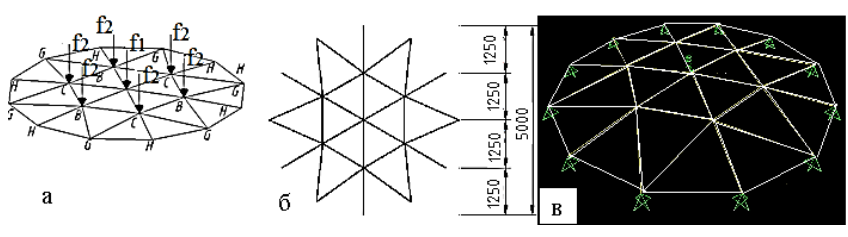

Рисунок 6 - Варианты задания перемещений узлов двухъярусного фрагмента сетчатого купола (а),

его размеры в плане (б) и общий вид расчётной модели (в)

Были произведены численные расчёты в программе SAP2000 с учётом геометрической нелинейности, полагая модуль упругости материала стержней постоянным (материал - сталь, E = 206000 Мпа) и не рассматривая иные формы потери устойчивости. В качестве аргумента при проведении численных расчётов принимались вертикальные перемещения узлов, найденные по изложенным выше зависимостям, их горизонтальные перемещения не ограничивались. Величины вертикальных перемещений узлов $f_{1}$ и $f_{2}$ приведены в таблице 4. Положительные величины $f_{1}$ и $f_{2}$ соответствует направления перемещений сверху вниз.

Таблица 4 - Вертикальные перемещения узлов, использованные при сравнении аналитического расчёта с численным

\begin{tabular}{|c|c|c|c|c|c|c|c|c|c|c|c|}
\hline \multicolumn{9}{|c|}{$n^{2}=1$} & \multicolumn{2}{c|}{$r=0$} & \multicolumn{2}{c|}{$r \rightarrow \infty$} & \multicolumn{2}{c|}{$r=10$} & \multicolumn{2}{c|}{$r=0$} \\
\hline \multicolumn{2}{|c|}{$r \rightarrow \infty=10$} & \multicolumn{2}{c|}{$r=5$} & $f 1$ & $f 1$ & $f 2$ \\
\hline$f 1$ & $f 2$ & $f 1$ & $f 2$ & $f 1$ & $f 2$ & $f 1$ & $f 2$ & $f 1$ & $f 2$ & $f 1$ \\
\hline 0.00 & 0.00 & 0.00 & 0.00 & 0.00 & 0.00 & 0.00 & 0.00 & 0.00 & 0.00 & 0.00 & 0.00 \\
\hline 5.80 & 3.80 & 5.84 & 3.84 & 2.95 & 0.95 & 13.95 & 9.45 & 14.83 & 10.33 & 6.65 & 2.15 \\
\hline 11.53 & 7.53 & 11.44 & 7.44 & 5.25 & 1.25 & 26.30 & 17.30 & 28.04 & 19.04 & 11.81 & 2.81 \\
\hline 17.10 & 11.10 & 16.98 & 10.98 & 7.17 & 1.17 & 37.40 & 23.90 & 39.29 & 25.79 & 16.14 & 2.64 \\
\hline 22.50 & 14.50 & 22.34 & 14.34 & 8.91 & 0.91 & 46.89 & 28.89 & 48.51 & 30.51 & 20.05 & 2.05 \\
\hline 27.91 & 17.91 & 27.64 & 17.64 & 10.57 & 0.57 & 55.13 & 32.63 & 56.05 & 33.55 & 23.79 & 1.29 \\
\hline 33.16 & 21.16 & 32.76 & 20.76 & 12.20 & 0.20 & 62.67 & 35.67 & 62.30 & 35.30 & 27.46 & 0.46 \\
\hline 38.41 & 24.41 & 37.88 & 23.88 & 13.85 & -0.15 & 69.31 & 37.81 & 67.68 & 36.18 & 31.15 & -0.35 \\
\hline 43.58 & 27.58 & 42.88 & 26.88 & 15.51 & -0.49 & 75.59 & 39.59 & 72.59 & 36.59 & 34.91 & -1.09 \\
\hline 48.75 & 30.75 & 47.94 & 29.94 & 17.22 & -0.78 & 81.70 & 41.20 & 77.36 & 36.86 & 38.75 & -1.75 \\
\hline 54.00 & 34.00 & 52.94 & 32.94 & 18.98 & -1.02 & 87.62 & 42.62 & 82.19 & 37.19 & 42.72 & -2.28 \\
\hline
\end{tabular}


Вестник Брестского государственного технического университета. 2021

С учётом изменяющихся углов наклона хорд стержней к горизонтальной плоскости были сопоставлены суммы вертикальных проекций продольных и поперечных сил в стержнях верхнего стержневого многогранника с результатами аналитического расчёта. Диаграммы равновесных состояний верхнего стержневого многогранника приведены на рисунке 7
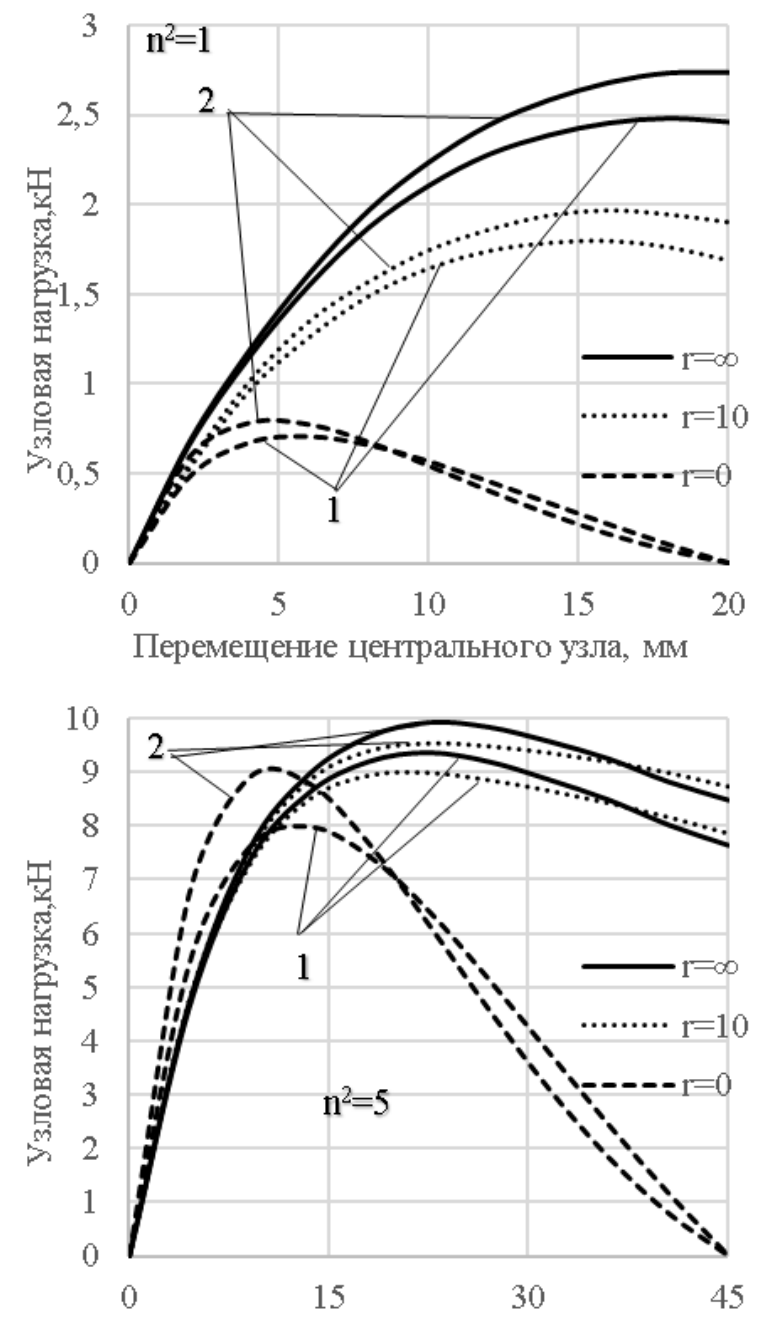

Перемещенне центрального узла, мм

1 - результаты аналитического расчёта,

2 - результаты численного расчёта

Рисунок 7 -Диаграммы равновесных состояний верхнего стержневого многогранника

Величины расхождеиий абсолютных величин ординат равновесных состояний при фиксированных значениях перемещений составляют 5-30 \%. При этом большей величине расхождений соответствуют меньшие значения ординат, кроме этого наблюдаются разные характеры десормирования стержневых многогранников в отдельных случаях (при $n^{2}=1$ и $r \rightarrow \infty$ диаграмма равновесных состояний, полученная при использовании численного метода расчёта не имеет критических точек, в отличие от одноимённой диаграммы, полученной аналитически). Следует также отметить, что величинь критических нагрузок, полученные с использованием численного метода расчёта, завышены относительно аналогичных значений, полученных по предложенным выше зависимостям. Также следует отметить, что в программных комплексах по расчёту конструкций зданий и сооружений отсутствует критерий наступления такого вида потери устойчивости. По этой причине при исследовании сетчатых куполов на потерю устойчивости, связанную с продавливанием их узлов к центру описанной сфреры, взяв за аргумент узловую нагрузку, получить диаграмму равновесных состояний с нисходящей ветвью невозможно: при достижении либо малейшем превышении значения критической нагрузки произойдёт переход от устойчивого выпуклого состояния стержневого многогранника к устойчивому вогнутому состоянию. При этом будет наблюдаться довольно значительное, скачкообразное приращение вертикальных перемещений узлов. При отсутствии в программных комплексах критериев достижения потери устойчивости такого вида крайне сложно обнаружить в каком месте в рассматриваемом сетчатом куполе будет достигнута (или не достигнута) величина критической узловой нагрузки, вызвавшая продавливание его узлов.

\section{Заключение}

1. Получены функции продольного изгиба стержня, зависящие от безразмерных параметров продольных сил и податливостей узлов, позволяющие вычислить величины опорных реакций стержней, упруго защемлённых по концам, при смещении или повороте упругих заделок.

2. Получены зависимости, описывающие процесс деформирования стержневых многогранников, образующих сетчатые купола с упруго-податливыми узлами, которые позволяют учитывать их геометрически нелинейное деформирование, продольно-поперечный изгиб и начальные геометрические несовершенства формы, жёсткость упругого защемления стержней в узлах, что даёт возможность вычислить величины критических узловых нагрузок.

3. Предложены коэффициенты для определения безразмерных параметров критических узловых нагрузок для выпуклых стержневых многогранников в виде правильных шестиугольников и пятиугольников в плане для различных величин их эквивалентной гибкости, неравномерности осесимметричного загружения и безразмерного параметра жёсткости узлов. Разработана и предложена методика проверки местной устойчивости сетчатых куполов с упруго-податливыми узлами. Сформулировано условие устойчивости их формы.

\section{Список цитированных источников}

1. Глушко, К. К. Влияние начальных несовершенств формы на местную устойчивость сетчатого купола при действии осесимметричной узловой нагрузки / К. К. Глушко // Актуальные проблемы исследования материалов, конструкций, технологий и организации строительства в трансграничном аспекте : сборник статей II Международной научной конференции, Брест, 18-20 октября 2017 г. / Брест. гос. техн. ун-т ; редкол.: С. М. Семенюк [и др.]. - Брест : БрГТУ, 2017. - С. 32-36.

2. Глушко, К. К. Исследование устойчивости формы стержневых многогранников сетчатых куполов / К. К. Глушко // Теория и практика исследований и проектирования в строительстве с применением систем автоматизированного проектирования (САПР) : сб. ст. II Междунар. науч.-техн. конф., Брест, 29-30 марта 2018 г. / Брест. гос. техн. ун-т ; редкол.: С. М. Семенюк [и др.]. - Брест : БрГТУ, 2018. - С. 24-33.

3. Глушко, К. К. Потеря местной устойчивости формы сетчатых куполов С жёсткими узлами / К. К. Глушко // Теория и практика исследований и проектирования в строительстве с применением систем автоматизированного проектирования (САПР) : сб. ст. II Междунар. науч.-техн. конф., Брест, 29-30 марта 2018 г. / Брест. гос. техн. ун-т ; редкол.: С. М. Семенюк [и др.]. - Брест : БрГТУ, 2018. - С. 33-42.

4. Глушко, К. К. Местная устойчивость стальных сетчатых куполов с шарнирным соединением стержней в узлах / К. К. Глушко, К. А. Глушко // Вестн. Брест. гос. техн. ун-та. - 2020. - № 1 : Строительство и архитектура. - С. 36-42.

5. Драган, В. И. Определение изгибающих моментов и поперечных сил в радиальной плоскости в стержнях сетчатого купола / В. И. Драган, К. К. Глушко // Теория и практика исследований и проектирования в строительстве с применением систем автоматизированного проектирования (САПР) : сб. ст. Междунар. науч.техн. конф., Брест, 30-31 марта 2017 г. / Брест. гос. техн. ун-т ; редкол.: С. М. Семенюк [и др.]. - Брест : БрГТУ, 2017. - С. 31-38. 
6. Драган, В. И. Определение продольных сил в стержнях и критических нагрузок, вызывающих местную потерю устойчивости сетчатого купола / В. И. Драган, К. К. Глушко // Теория и практика исследований и проектирования в строительстве с применением систем автоматизированного проектирования (САПР) : сб. ст. Междунар. науч.-техн. конф., Брест, 30-31 марта 2017 г. / Брест. гос. техн. ун-т ; редкол.: С. М. Семенюк [и др.]. - Брест : БрГТУ, 2017. - C. 39-46.

7. Журавлев, А. А. К вопросу о местной устойчивости сетчатых куполов с треугольной решеткой / А. А. Журавлев // Изв. вузов. Строительство и архитектура. - 1971. - № 5. - С. 77-80.

8. Журавлев, А. А. Пространственные деревянные конструкции А. А. Журавлев, Г. Б. Вержбовский, Н. Н. Ерёменко. - Ростов н/Д : РГСУ, 2003. $-518 \mathrm{C}$

9. Журавлев, А. А. Устойчивость стержневых систем в форме выпуклых конфигураций на плоскости и в пространстве / А. А. Журавлев // Известия РГАС. - 1996. - № 1. - С. 42-48.

10. Коршун, Л. И. Основы устойчивости стержневых систем / Л. И. Коршун, В. И. Игнатюк, А. С. Хамутовский. - Брест, 1995. - 64 с.

11. Тур, В. И. Влияние податливости узловых соединений на напряженно-деформированное состояние металлического сетчатого купола / В. И. Тур, А. В. Тур // Фундаментальные исследования. 2014. - № 6 : Технические науки. - С. 1165-1168.

12. Abedi, K. Progressive collapse of single-layer braced domes / K. Abedi, G.A.R. Parke // International Journal of Space Structures. 1991. - № 11 (3). - P. 291-306.

13. Abedi, K. Propagation of local instabilities in braced domes / K. Abedi : doctoral dissertation. - University of Surrey, 1997. - $333 p$

14. Gioncu, V. Buckling of Reticulated Shells: State-of-theArt / V. Gioncu /I International Journal of Space Structures. - 1995. - Vol. 10, № 1. P. 1-46.

15. Kato, S. Dynamic Elasto-Plastic Buckling Simulation System for Single Layer Reticular Domes with Semi-Rigid Connections under Multiple Loadings / S. Kato, M. Murata // International Journal of Space Structures. - 1997. - Vol.12, № 3-4. - P. 161-172.

16. Lopez, A. Numerical model and experimental tests on single-layer latticed domes with semi-rigid joints / A. Lopez, I. Puente, M. A. Serna // Computers and Structures. - 2007. - № 85. - P. 360-374.

\section{References}

1. Hlushko, K. K. Vliyanie nachal'nyh nesovershenstv formy na mestnuyu ustojchivost' setchatogo kupola pri dejstvii osesimmetrichnoj uzlovoj nagruzki / K. K. Hlushko // Ak-tual'nye problemy issledovaniya materialov, konstrukcij, tekhnologij i organizacii stroitel'stva $v$ transgranichnom aspekte : sbornik statej II Mezhdunarodnoj nauchnoj konferencii, Brest, 18-20 oktyabrya 2017 g. / Brest. gos. tekhn. un-t ; redkol.: S. M. Seme-nyuk [i dr.]. - Brest : BrGTU, 2017. - S. 32-36.

2. Hlushko, K. K. Issledovanie ustojchivosti formy sterzhnevyh mnogogrannikov set-chatyh kupolov / K. K. Hlushko // Teoriya i praktika issledovanij i proektirovaniya $v$ stroitel'stve s primeneniem sistem avtomatizirovannogo proektirovaniya (SAPR) : sb. st. II Mezhdunar. nauch.tekhn. konf., Brest, 29-30 marta 2018 g. / Brest. gos. tekhn. un-t ; redkol.: S. M. Semenyuk [i dr.]. - Brest : BrGTU, 2018. -S. 24-33.

3. Hlushko, K. K. Poterya mestnoj ustojchivosti formy setchatyh kupolov s zhyostkimi uzlami / K. K. Hlushko // Teoriya i praktika issledovanij i proektirovaniya $v$ stroitel'-stve $s$ primeneniem sistem avtomatiziro- vannogo proektirovaniya (SAPR) : sb. st. II Mezhdunar. nauch.tekhn. konf., Brest, 29-30 marta 2018 g. / Brest. gos. tekhn. un-t ; redkol.: S. M. Semenyuk [i dr.]. - Brest : BrGTU, 2018. - S. 33-42.

4. Hlushko, K. K. Mestnaya ustojchivost' stal'nyh setchatyh kupolov $\mathrm{s}$ sharnirnym so-edineniem sterzhnej $\mathrm{v}$ uzlah / K. K. Hlushko, K. A. Glushko // Vestn. Brest. gos. tekhn. un-ta. - 2020. - № 1 : Stroitel'stvo i arhitektura. - S. 36-42.

5. Dragan, V. I. Opredelenie izgibayushchih momentov i poperechnyh sil v radial'noj ploskosti v sterzhnyah setchatogo kupola / V. I. Dragan, K. K. Hlushko // Teoriya i praktika issledovanij i proektirovaniya v stroitel'stve $s$ primeneniem sistem avtomatiziro-vannogo proektirovaniya (SAPR) : sb. st. Mezhdunar. nauch.-tekhn. konf., Brest, 30-31 marta 2017 g. / Brest. gos. tekhn. un-t ; redkol.: S. M. Semenyuk [i dr.]. - Brest : BrGTU, 2017. - S. 31-38

6. Dragan, V. I. Opredelenie prodol'nyh sil v sterzhnyah i kriticheskih nagruzok, vy-zyvayushchih mestnuyu poteryu ustojchivosti setchatogo kupola / V. I. Dragan, K. K. Hlushko // Teoriya i praktika issledovanij i proektirovaniya $v$ stroitel'stve $s$ primeneniem si-stem avtomatizirovannogo proektirovaniya (SAPR) : sb. st. Mezhdunar. nauch.tekhn. konf., Brest, 30-31 marta 2017 g. / Brest. gos. tekhn. un-t ; redkol.: S. M. Semenyuk [i dr.]. - Brest : BrGTU, 2017. - S. 39-46.

7. Zhuravlev, A. A. K voprosu o mestnoj ustojchivosti setchatyh kupolov s treugol'noj reshetkoj / A. A. Zhuravlev // Izv. vuzov. Stroitel'stvo i arhitektura. - 1971. - № 5. - S. 77-80.

8. ZHuravlev, A. A. Prostranstvennye derevyannye konstrukcii / A. A. Zhuravlev, G. B. Verzhbovskij, N. N. Eryomenko. - Rostov n/D : RGSU, 2003. $-518 \mathrm{~s}$.

9. Zhuravlev, A. A. Ustojchivost' sterzhnevyh sistem v forme vypuklyh konfiguracij na ploskosti i v prostranstve / A. A. Zhuravlev // Izvestiya RGAS. - 1996. - № 1. - S. 42-48.

10. Korshun, L. I. Osnovy ustojchivosti sterzhnevyh sistem / L. I. Korshun, V. I. Ignatyuk, A. S. Hamutovskij. - Brest, 1995. - 64 s.

11. Tur, V. I. Vliyanie podatlivosti uzlovyh soedinenij na napryazhennodeformirovannoe sostoyanie metallicheskogo setchatogo kupola 1 V. I. Tur, A. V. Tur // Fundamental'nye issledovaniya. - 2014. - № 6 : Tekhnicheskie nauki. - S. 1165-1168.

12. Abedi, K. Progressive collapse of single-layer braced domes / K. Abedi, G.A.R. Parke // International Journal of Space Structures. 1991. - № 11 (3). - P. 291-306.

13. Abedi, K. Propagation of local instabilities in braced domes / K. Abedi : doctoral dissertation. - University of Surrey, 1997. - 333 p.

14. Gioncu, V. Buckling of Reticulated Shells: State-of-theArt / V. Gioncu // International Journal of Space Structures. - 1995. - Vol. 10, № 1. P. 1-46.

15. Kato, S. Dynamic Elasto-Plastic Buckling Simulation System for Single Layer Reticular Domes with Semi-Rigid Connections under Multiple Loadings / S. Kato, M. Murata // International Journal of Space Structures. - 1997. - Vol.12, № 3-4. - P. 161-172.

16. Lopez, A. Numerical model and experimental tests on single-layer latticed domes with semi-rigid joints / A. Lopez, I. Puente, M. A. Serna // Computers and Structures. - 2007. - № 85. - P. 360-374.

Материал поступил в редакцию 21.12.2020 